\title{
Modélisation de la distribution spatiale de la girafe (Giraffa camelopardalis peralta, Linnaeus 1758) de l'Afrique de l'Ouest pour sa conservation au Niger
}

\author{
O. HAMADOU ${ }^{*}$, A. AMADOU OUMANI ${ }^{1}$, H. YAHOU ${ }^{2}$, B. MOROU ${ }^{1}$ et \\ A. MAHAMANE ${ }^{2,3}$
}

\author{
${ }^{1}$ Université Dan Dicko Dankoulodo de Maradi, BP 465 Maradi, Niger. \\ ${ }^{2}$ Université de Diffa, BP 78, Diffa, Niger.
}

${ }^{3}$ Université Abdou Moumouni de Niamey, BP 1066 Niamey, Niger.

*Auteur correspondant ; E-mail : omarita10@yahoo.fr, Tel : (00227) 96471800

\begin{abstract}
Received: 14-07-2021
Accepted: 04-12-2021

Published: 31-12-2021

\section{RESUME}

La conservation de la girafe (Giraffa camelopardalis peralta, Linnaeus 1758), nécessite la connaissance de son aire de répartition potentielle et des facteurs environnementaux conditionnant cette répartition. Cette étude visait à identifier les facteurs écologiques régissant la répartition géographique de la girafe et la cartographie de son aire de distribution sous les conditions climatiques actuelles pour une meilleure planification de sa gestion durable. La modélisation a été utilisée pour cartographier l'habitat de la girafe suivant les modèles climatiques (CCMA et CSIRO), à l'aide de l'outil Maxent, Diva-Gis et ArcGi 10.3. Cinq variables bioclimatiques (BIO1, BIO 12, BIO 13, BIO3 et BIO 5) ont été sélectionnées sur les 19 variables existantes, elles ont ensuite été utilisées avec les points d'occurence de la girafe dans l'outil Maxent. Les variables les plus significatives conditionnant la distribution de la girafe étaient les précipitations et les températures annuelles. Les distributions futures (2020 et 2050) ont montré des habitats très favorables, avec une augmentation de 21,22 à 59,95\% par rapport à la distribution actuelle. Des mesures d'atténuer la dégradation continue de de l'habitat de la girafe s'imposent en vue de maintenir sa dynamique actuelle et permettre sa conservation. Aussi, il serait intéressant de mettre en place un protocole du suivi permanent des comportements de la girafe, y compris ses mouvements saisonniers afin d'anticiper les sorties vers des zones hostiles pour la conservation.
\end{abstract}

(C) 2021 International Formulae Group. All rights reserved.

Mots clés : Modélisation, Distribution, Habitat, Conservation, Girafe.

\section{Modelling the spatial distribution of the West African giraffe (Giraffa camelopardalis peralta, Linnaeus 1758) for its conservation in Niger}

\begin{abstract}
The conservation of the giraffe (Giraffa camelopardalis peralta, Linnaeus 1758) requires knowledge of its potential range and the environmental factors conditioning this distribution. This study aimed to identify the ecological factors governing the geographic distribution of the giraffe and to map its range under current climatic conditions for better planning of its sustainable management. Modeling was used to map giraffe habitat under climate models (CCMA and CSIRO), using Maxent, Diva-Gis and ArcGi 10.3. Five bioclimatic variables (BIO1,
\end{abstract}


BIO 12, BIO 13, BIO3 and BIO 5) were selected from the 19 existing variables and used with giraffe occurrence points in Maxent. The most significant variables conditioning the giraffe distribution were annual precipitation and temperature. Future distributions (2020 and 2050) showed very favorable habitats, with an increase of 21.22 to $59.95 \%$ over the current distribution. Measures to mitigate the continued degradation of the giraffe's habitat are needed to maintain its current dynamics and allow its conservation. Also, it would be interesting to set up a protocol for the permanent monitoring of the giraffe's behaviors, including its seasonal movements in order to anticipate the exits towards hostile areas for the conservation.

(C) 2021 International Formulae Group. All rights reserved.

Keywords : Modeling, Distribution, Habitat, Conservation, Giraffe.

\section{INTRODUCTION}

Au cours des dernières décennies, la population Ouest Africaine a connu une importante croissance démographique (Hamadou, 2014). L'extension des zones agricoles, l'élevage, le déboisement, la chasse et les modifications du climat ont très largement contribué et contribuent encore à la diminution et à la fragmentation des espaces naturels (Cornelis, 2011). A telle enseigne qu'aujourd'hui, la grande faune sauvage se trouve confinée à l'intérieur d'aires dites « protégées », dont la plupart ne le sont que de nom (Joppa, 2008). Au Niger, l'aire de distribution de la girafe a fortement régressé, d'une part en raison de la fragmentation des milieux naturels par l'Homme, et d'autre part $\mathrm{du}$ fait des conditions et variabilités climatiques (Morou, 2010).

L'habitat de la girafe constitue l'un des écosystèmes naturels des savanes au Niger qui abrite une population de girafes mobile en fonction des saisons. En septembre 2006, une contribution à l'élaboration d'une stratégie de conservation à long terme de la girafe (Giraffa camelopardalis peralta) au Niger de cette aire écologique a été mise en place par le Programme Régional Parc W/ECOPAS (Ecosystème Protégés en Afrique SoudanoSahélienne), financé par les Fonds Européens de Développement, l'Etat du Niger, avec le soutien significatif des ONG locales pour promouvoir la conservation, et la protection de la girafe et de son habitat.

Toutefois, les informations sur les déterminants écologiques de l'utilisation de l'espace, du déplacement et de ses exigences en termes d'habitat sont peu nombreuses pour caractériser la distribution de la girafe. Pour préciser l'aire potentielle de distribution des espèces, il est nécessaire d'étudier la gamme des conditions environnementales qui lui convient (Guisan, 2000) et de procéder sur cette base à la modélisation de sa distribution potentielle. Ces informations permettront de connaître les exigences de la girafe en termes d'habitats et d'interactions avec l'environnement. Elles permettront également de mettre en œuvre les politiques de conservation de la girafe de façon efficace et efficiente. Ainsi, émergeront des solutions permettant d'atténuer les conflits entre l'Homme et la girafe (qui détruit les cultures). La girafe est une source de devises importantes pour les communautés locales et l'Etat du Niger. Les écosystèmes de la zone girafe sont soumis à des pressions d'ordre socioéconomiques des populations rurales qui les exploitent (Benadid, 2000). Ils sont aussi liés à l'aridification croissante observée (IPPC, 2014) et la sensibilité des terres à la désertification (MDCE, 2016). Ces menaces ont eu pour conséquence des réductions des aires géographiques favorables, et de la disponibilité des habitats pour certaines espèces forestières. Cette situation compromet les efforts des conservateurs et gestionnaires des ressources forestières qui doivent souvent œuvrer avec des moyens limitées pour restaurer et gérer durablement les écosystèmes forestiers (Moukrim, 2018).

Le déplacement des herbivores relève d'interactions multiples. La satisfaction des besoins alimentaires est vitale, et fortement conditionnée par le type, la densité, et l'agencement spatial des ressources (biotiques et abiotiques). Les herbivores choisissent les habitats de façon à maximiser leur gain 
énergétique net et optimiser ainsi leurs chances de survie en matière alimentaire et de reproduction. Le risque de prédation est également une force sélective importante, conditionnant potentiellement les stratégies de déplacement (Ripple et al., 2004 ; Creel et al., 2005).

La Modélisation de la Distribution des Espèces (SDM pour Species Distribution Modeling) représente un outil important de prédiction en écologie de la conservation (Guisan et al., 2000 ; Fleishman et al., 2001 ; Scott et al., 2002 ; Ripple et al., 2004 ; Elith et al., 2006 ; Austin, 2007 ; Frankline, 2009; Peterson et al., 2012; Padalia et al., 2014). L'objectif de cette étude était de modéliser en vue d'identifier l'aire de distribution potentielle de la girafe ainsi que les variables environnementales affectant sa répartition géographique.

\section{MATERIEL ET METHODES}

Le principe d'Entropie Maximale (Maxent), a été utilisé dans le cadre de cette étude.

La Modélisation de la Distribution des Espèces (SDM pour Species Distribution Modeling) représente un outil important de prédiction en écologie et d'aide à la conservation de la conservation de la biodiversité (Guisan et al., 2000 ; Fleishman et al., 2001 ; Scott et al., 2002 ; Ripple et al., 2004 ; Elith et al., 2006 ; Austin, 2007 ; Frankline, 2009 ; Peterson et al., 2012 ; Padalia et al., 2014). Elle consiste à construire une fonction de paramètres environnementaux qui prédit la probabilité de présence et permettra de mettre en évidence les habitats favorables actuels et futurs de la girafe. Le jeu de données utilisé comprend des données de présence actuelle de l'espèce et des valeurs de certains paramètres, essentiellement environnementaux (Phillips et al., 2004; Elith et al. 2006 ; Sampoux et al., 2009). Plusieurs méthodes pour modéliser la distribution des espèces ont été développées et utilisées (Phillips, 2004), elles diffèrent suivant le type de réponse qu'elles prennent en compte, la manière de pondérer les observations et d'incorporer les interactions, et suivant leur capacité de prédiction (Elith et al., 2006 ; Elith et al., 2009; Moukrim, 2018). Aujourd'hui, les modèles de distribution d'espèces sont de plus en plus utilisés pour répondre aux problématiques majeures telles que la compréhension, la description et la prédiction de l'aire potentielle d'une espèce et l'identification des facteurs qui déterminent sa distribution (Kumar, 2009). Ils représentent de ce fait, un outil pertinent pour la gestion et la conservation de l'environnement et de la biodiversité (Guisan et al., 2000 ; Barbasa et al., 2009).

Le test de Jackknife (Miller, 1974) a été aussi effectué sur les variables considérées afin de déterminer le pouvoir prédictif de chaque variable et d'identifier celles qui contribuent le plus au modèle. Des tests de Jackknife ont été exécutés sur les variables climatiques pour déterminer les grands contributeurs au modèle de prédiction.

\section{Présentation de la zone d'étude}

Cette étude a été conduite dans la zone de distribution de la girafe entre $12^{\circ} 00^{\prime}$ et $14^{\circ} 00^{\prime}$ de latitude Nord et $2^{\circ} 00^{\prime}$ à $5^{\circ} 00^{\prime}$ de longitude Est. Elle est à cheval entre trois (3) régions (Tillabéri, Dosso et Tahoua) et couvre près de $83000 \mathrm{~km}^{2}$ de superficie (Figure 1). Sur le plan géomorphologique, il est rencontré :

- les sols du plateau ou Fakara qui sont des mélanges de sables éoliens et des produits argilo-sableux issus $\mathrm{du}$ continental terminal et les sols des agrosystèmes qui sont en voie de dégradation poussée par la déflation et le ruissellement (Ambouta, 1999).

- La brousse tigrée qui est une formation forestière constituée d'alternance de bandes de végétation et de sol nu. Les processus qui déterminent son évolution ont été relativement investigués. C'est l'une des formations végétales la mieux structurée de la zone sahélienne (Ambouta, 1999).

Les Végétations des agrosystèmes sont extrêmement dégradées. On retrouve au niveau des reliquats de brousse tigrée, une végétation typique de la formation du plateau (Hamadou, 
2009). La forêt de Gorou Bassounga est classée depuis 1935 et est située en zone soudanienne, elle se caractérise par une forte densité d'arbres et une grande variété d'espèces végétale. La région fréquentée par les girafes à l'Ouest du village d'Ouna est une zone du Dallol Bosso Sud située en zone nord soudanienne. C'est une zone cultivée à proximité du fleuve Niger (Ciofolo, 1998). Les pluies sont irrégulières et mal réparties dans le temps et dans l'espace et avec une pluviométrie moyenne annuelle de 497,15 mm enregistrée ces 30 dernières années, dans la zone de Gaya, cette moyenne peut atteindre $700 \mathrm{~mm}$. Trois saisons alternent au cours de l'année, la saison sèche froide, la saison sèche chaude et la saison pluvieuse : la saison des pluies (3 à 4 mois, c'est-à-dire de Juillet à Octobre), pendant laquelle les girafes restent sur les plateaux forestiers; les saisons sèches froide et sèche chaude de Novembre à Juin, au cours desquelles les girafes sont dans les agrosystèmes. Les maximas des températures passent de $34,2^{\circ} \mathrm{C}$ en fin de saison sèche chaude (avril) à $30,7^{\circ} \mathrm{C}$ à la fin de la saison des pluies (septembre et octobre) et les minimas gravitent autour de $23,2^{\circ} \mathrm{C}$ pendant la saison sèche froide (décembre) et $27,7^{\circ} \mathrm{C}$ en pleine saison des pluies, août, (Ambouta, 1994).

\section{Collecte des données}

Positions géographiques

Les coordonnées géographiques utilisées ont été collectées pendant les différentes opérations de dénombrements d'une part et celles issues de nos visites de terrain lors du suivi écologique d'autre part.

\section{Variables environnementales}

Elles proviennent de la base des données worldclim (www.worldclim.org) à une haute résolution spatiale pour le climat global et les données climatiques. Elle est utilisée pour la modélisation spatiale dans le domaine de la recherche et de certaines activités (Hijmans, 2005). Les données sont extraites des fichiers à extension.clm constituées de 19 variables bioclimatiques ou environnementales à base essentiellement des températures et précipitations (Tableau 1).
Les données actuelles couvrent la période de 1950 à 2000 avec une résolution spatiale de $2.5 \mathrm{mn}$ (soit $5 \mathrm{~km} \mathrm{x} 5 \mathrm{~km}=25 \mathrm{~km}^{2}$ ) (www.worldclim.org). Les données futures couvrent quant à elles, la période de 2020 à 2050.

\section{Analyse des données}

Les 19 variables (bioclimatiques et environnementales) ont été chargées sur DIVA GIS 7-5 pour générer les variables climatiques ascii qui sont des rasters pouvant être pris en charge (Phillips, 2004) par le logiciel de modélisation d'Entropie Maximale (MAXENT 3.3.3k). Ce programme a été choisi car il constitue l'une des meilleures méthodes de modélisation de la distribution des espèces, et qu'il est connu pour générer des modèles fiables (Baldwin, 2009). Il ne nécessite que peu de points de présence de l'espèce pour construire des modèles utiles (Phillips et al., 2004 ; Feeley et al., 2011) et il est relativement insensible aux différents biais spatiaux (Elith et al., 2006 ; Guisan et al., 2007). Ces variables sont extraites dans la limite de la couche shapefile de la zone à modéliser. Elles ont subi ensuite une série de transformations qui les ont faits évoluer des fichiers à extension.clm aux fichiers GRI et GRD. Un test de corrélation de Pearson est effectué pour sélectionner les variables les plus pertinentes pour le modèle. Ainsi les variables avec un coefficient de corrélation élevé ( $|\mathrm{r}|>0.7)$ et à importance écologique pour les espèces étudiées (Dormann, 2013) ont été identifiées. Seules les variables les moins corrélées sont chargés sur MAXENT pour générer le fichier raster des distributions des espèces. Le modèle climatique a été exécuté avec les cinq variables bioclimatiques ou environnementales retenues pour la distribution actuelle qui correspond à la période 1950 à 2000. Aussi, il a été gardé le même système de classification avec les classes «Très favorable », « Favorable », « Moyennement favorable » et «Peu favorable». Pour déterminer les variables qui contribuent le plus à la modélisation, le test de Jacknife a été effectué (Fandohan et al.,2013; Abdou, 2016). Trois répétitions ont été faites pour produire des estimations fiables. 
L'analyse AUC ou Area Under Curve évalue la performance du modèle. La validité intrinsèque d'un modèle est caractérisée par la sensibilité et la spécificité. Ces deux paramètres ont permis l'établissement de la Courbe Caractéristique d'Opération dite ROC (Receiving Operator Characteristic) (Hanley, 1982). Le calcul de l'AUC (aire sous la courbe ROC), qui est un seuil qui mesure la précision de la prédiction du modèle (, renseigne sur la performance et la qualité du modèle établi à prédire correctement l'occurrence de l'espèce (Fawett, 2006). Un modèle est dit excellent lorsque la valeur de l'AUC est supérieure à
0,90 ; il est bon lorsque $0,90>$ AUC $>0,80$; acceptable lorsque $0,80>$ AUC > 0,70, mauvais lorsque $0,70>$ AUC $>0,60$ et invalide $0,60>$ AUC > 0,50 (Phillips et al., 2004). Les modèles utilisés sont le CSIRO Mk3.5 (Commonwealth Scientific and Industrial Research Organisation) qui envisage un léger réchauffement de $0,2^{\circ} \mathrm{C}$ de la surface des mers sur une période allant sur 1300 ans et une diminution des précipitations et le modèle CCMA (Canadian Centre for Climate Modelling And Analysis) qui envisagent une augmentation des précipitations.

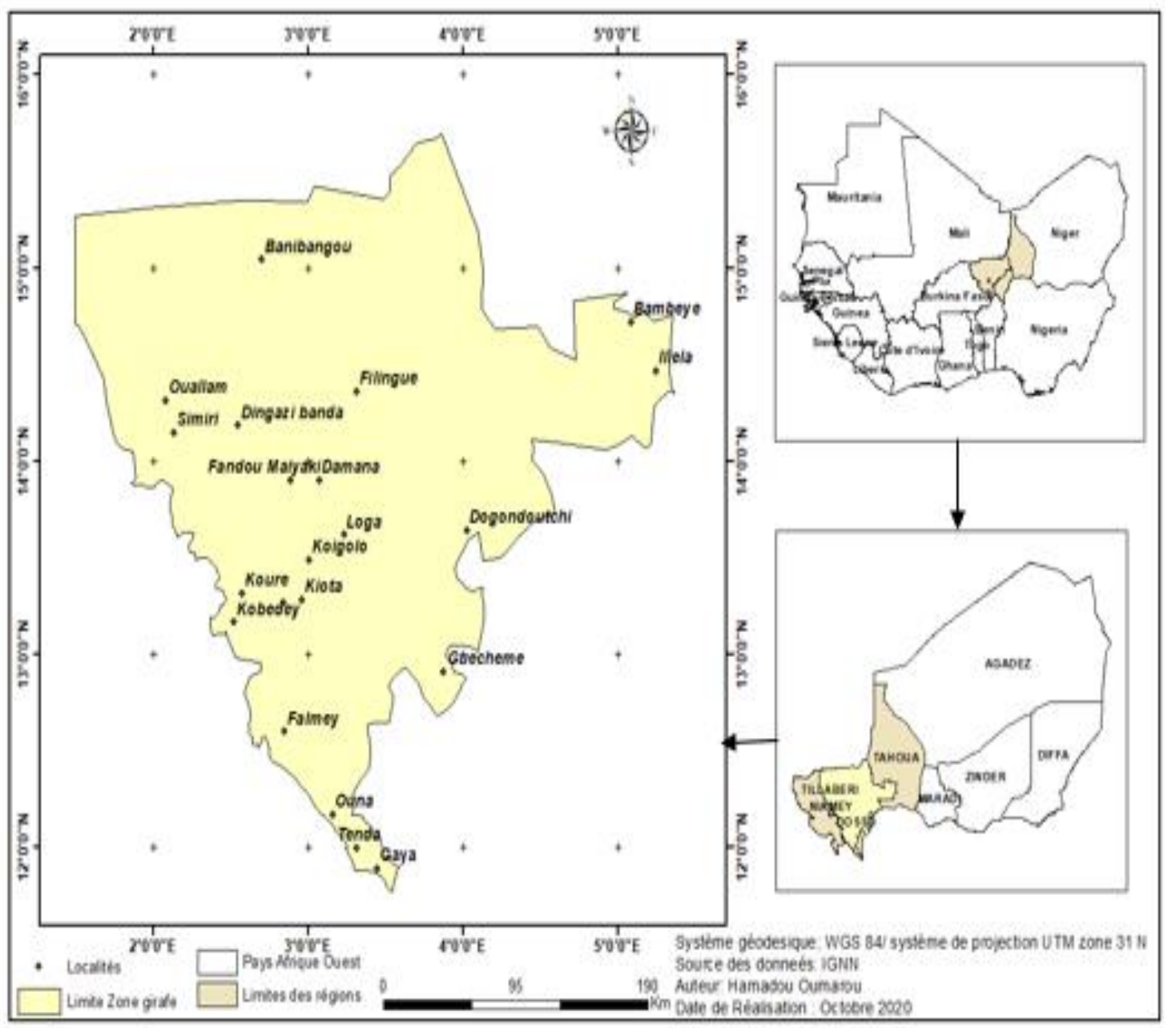

Figure 1 : Carte de localisation de la zone d'étude. 
Tableau 1 : Variables bioclimatiques ou environnementales utilisées pour la modélisation.

\begin{tabular}{ll}
\hline Code & Signification \\
\hline BIO1 & Température moyenne annuelle \\
BIO2 & Ecart diurne moyen (température maximale - température minimale ; moyenne mensuelle) \\
BIO3 & Isothermalité (BIO2/BIO7) *100 \\
BIO4 & Saisonnalité de la température (Coefficient de variation) \\
BIO5 & Température maximale de la période la plus chaude \\
BIO6 & Température minimale de la période la plus froide \\
BIO7 & Ecart annuel de température (BIO5-BIO6) \\
BIO8 & Température moyenne du trimestre le plus humide \\
BIO9 & Température moyenne du trimestre le plus sec \\
BIO10 & Température moyenne du trimestre le plus chaud \\
BIO11 & Température moyenne du trimestre le plus froid \\
BIO12 & Précipitations annuelles \\
BIO13 & Précipitations de la période la plus humide \\
BIO14 & Précipitations de la période la plus sèche \\
BIO15 & Saisonnalité des précipitations (Coefficient de variation) \\
BIO16 & Précipitations du trimestre le plus humide \\
BIO17 & Précipitations du trimestre le plus sec \\
BIO18 & Précipitations du trimestre le plus chaud \\
BIO19 & Précipitations du trimestre le plus froid \\
\hline
\end{tabular}

\section{RESULTATS}

\section{Distributions actuelles de la girafe}

Cinq (5) variables environnementales ont été retenues après le test de corrélation parmi les 19 variables disponibles pour le modèle Maxent (Figure 2). Il s'agit des variables BIO1 (Température moyenne annuelle), BIO12 (Précipitations annuelles), BIO13 (Précipitations de la période la plus humide), BIO3 [Isothermalité (BIO2/BIO7) *100] et BIO5 (Température maximale de la période la plus chaude). La variable «Bio 12 » est le prédicteur environnemental qui présente le meilleur gain et qui semble avoir l'information la plus utile lorsque cette variable est utilisée isolément dans le modèle. L'AUC ou Area Under Curve qui a permis d'apprécier le modèle est comprise entre 0,70 et 0,989 . Le modèle est par conséquent excellent.

\section{Qualité du Modèle et Variables Influentes}

La Figure 3 montre que la valeur de l'aire sous la courbe (AUC) pour la mise en œuvre du modèle MaxEnt est de 0,98. Ceci indique la robustesse de la modélisation effectuée et atteste l'excellente performance de l'algorithme MaxEnt à prédire l'aire favorable de l'espèce étudiée.

Le fichier raster issu du modèle a ensuite été traité dans le logiciel ArcGis 10.3. C'est une image qui nécessite une classification supervisée. Après la classification du raster issue de la période actuelle (1950 à 2000), différents habitats de la girafe (Très favorable, Favorable, Moyennement favorable et peu favorable), ont été mis en évidence (Figure 4).

Entre 1950 à 2000, sur une superficie totale de $83480,17 \mathrm{~km}^{2}$, la girafe occupe un 
habitat peu favorable de $24 \quad 456,81 \mathrm{~km} 2$ $(29,29 \%)$, moyennement favorable de $21645,14 \mathrm{~km}^{2}(25,92 \%)$, un habitat favorable de $19659,42 \mathrm{~km}^{2}$, soit $23,54 \%$ et un habitat très favorable de $17718,81 \mathrm{~km}^{2}$, soit $21,22 \%$.

\section{Distributions futures de la girafe Modèle climatique CCMA}

Le modèle climatique CCMA exécuté dans MaxEnt pour la distribution de la girafe a permis de déterminer leurs habitats pour les horizons 2020 (a) et 2050 (b). Il a été caractérisé par une valeur d'AUC supérieure à 0,90 , prouvant ainsi l'excellence du modèle pour cette espèce.

L'analyse des Figures a montré une perte de superficies des habitats peu favorable, moyennement favorable et favorable en 2020. Dans la même période, on a constaté une augmentation de l'habitat très favorable. L'horizon 2050 a montré selon des habitats «peu favorable» et « très favorable », relativement réduits et une légère augmentation des habitats «moyennement favorable» et « favorable ».

\section{Modèle climatique CSIRO}

Un second modèle dénommé CSIRO a été utilisé et est également excellent. Cette performance du modèle est due aux valeurs des AUC enregistrées, qui sont supérieures à 0,90 pour la girafe en horizon 2020 (c) et 2050 (d).
L'analyse des figures issues de ce modèle montre globalement pour tous les deux horizons (2020 et 2050) des habitats très favorables (Figure 7) plus importants pour la girafe. Les superficies des habitats «peu favorables », «moyennement favorables » et « favorables » étaient réduites dans l'ensemble de l'aire de répartition de la girafe suivant le modèle CSIRO.

Effets des changements climatiques sur l'habitat de la girafe

Les conditions climatiques caractérisées par des faibles précipitations et des amplitudes thermiques élevées ont entrainé une aridification du climat entraînant du coup la disparition ou la réduction de beaucoup d'espèces floristiques consommées par la girafe. Actuellement, la girafe occupe des nouvelles zones dans son aire de répartition. Les habitats actuels et futurs, issus des modèles CCMA et CSIRO, ont connu des variations entre les modèles et les périodes. Cependant l'habitat de la girafe semble être plus résilient aux changements climatiques, avec l'augmentation des superficies des classes très favorables. En effet, les modèles montrent un accroissement des superficies des classes « très favorables ». Les autres classes «peu favorables », « moyennement favorables » et «favorable », ont diminué, laissant la place à la classe très favorable (Tableau 2).

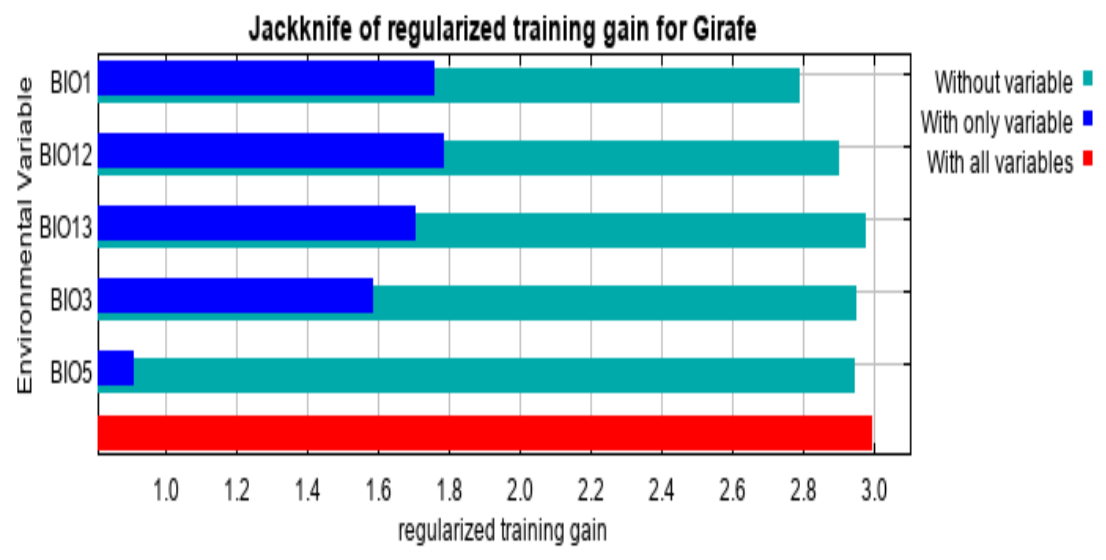

Figure 2 : Résultats du test de Jackknife sur les 5 variables retenues pour la girafe. 


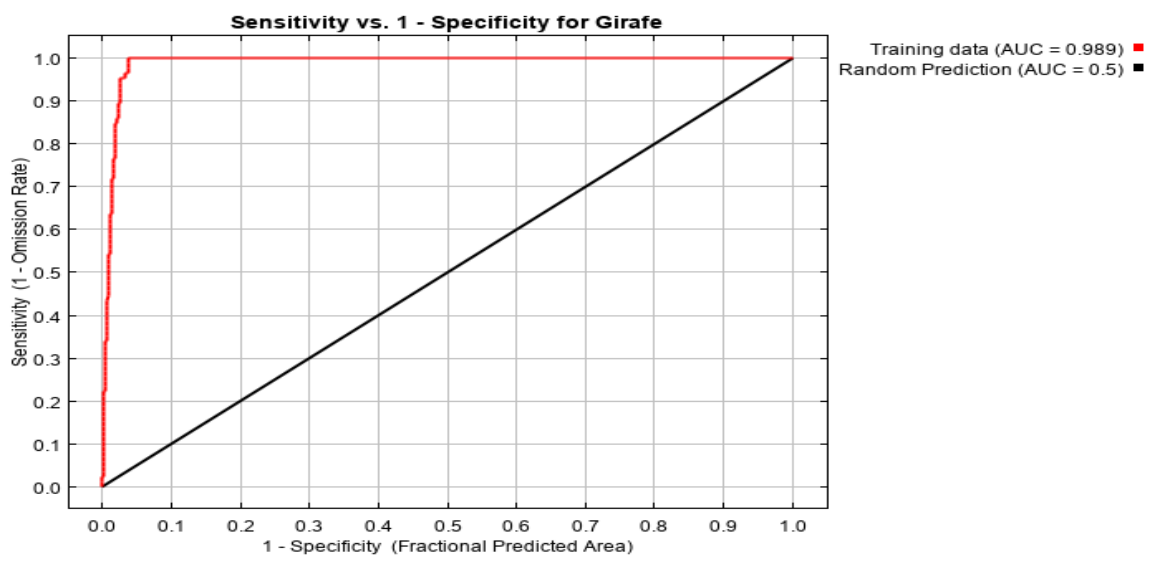

Figure 3: Résultat du calcul de l'AUC, aire sous la courbe caractéristique d'opération (ROC).

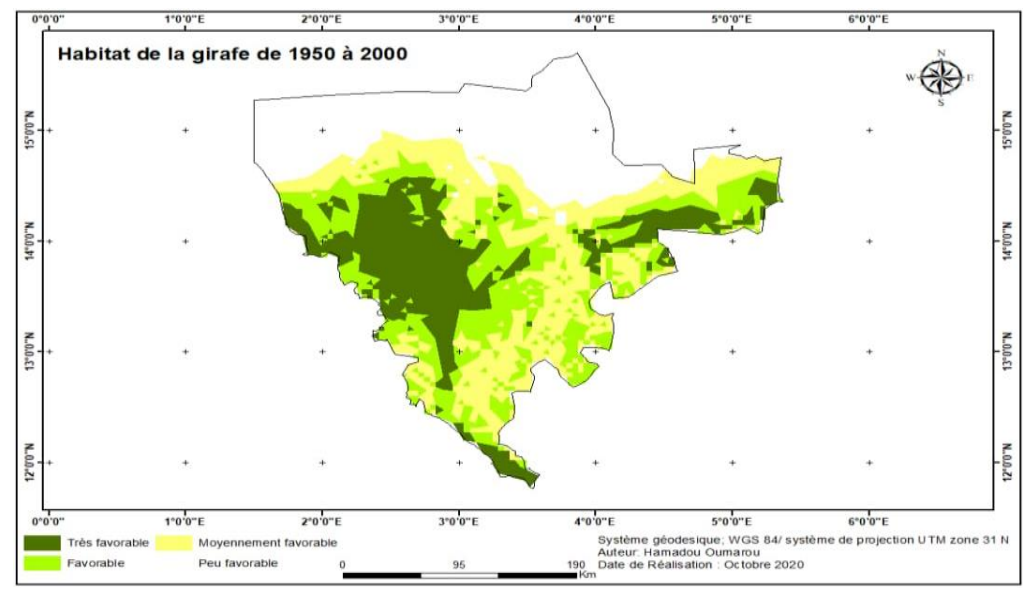

Figure 4: Habitat actuel de la girafe de 1950 à 2000.

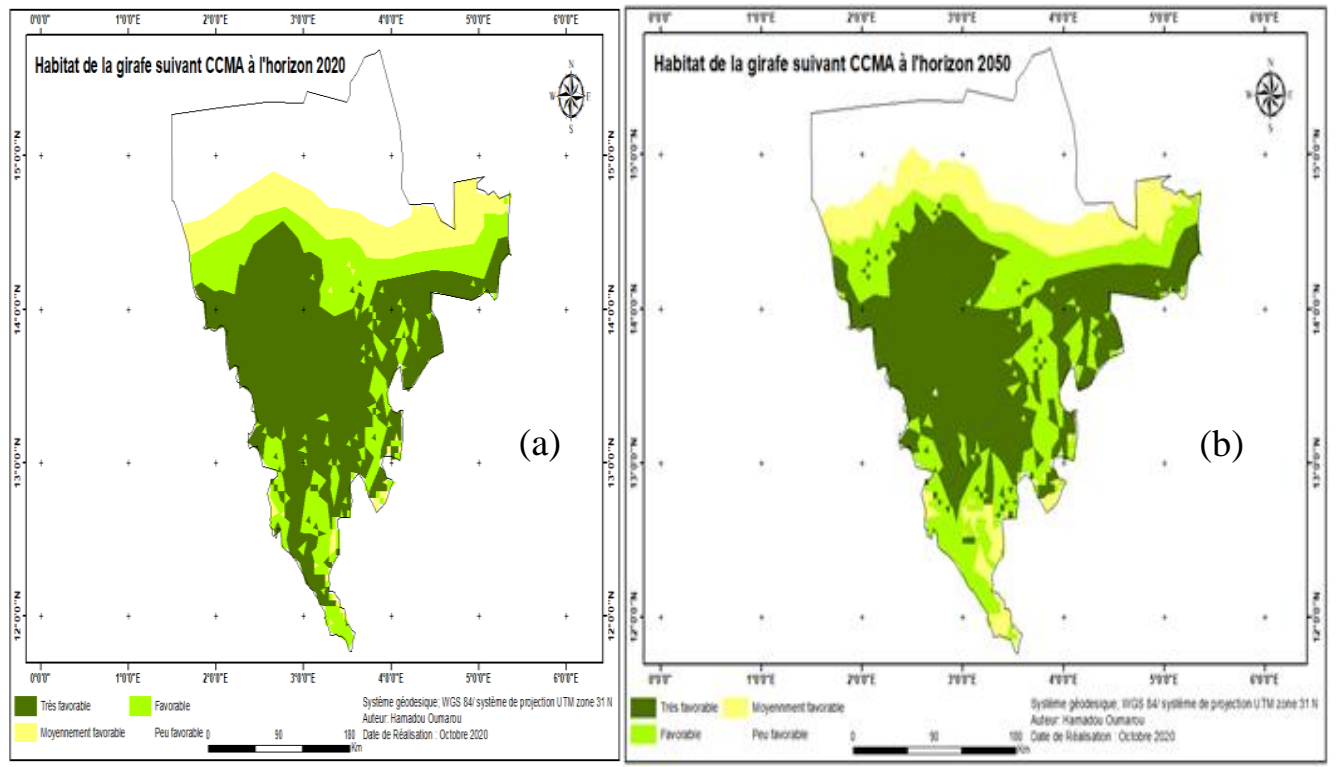

Figure 5: Habitat de la girafe suivant les modèles CCMA 2020 (a) et CCMA 2050 (b). 


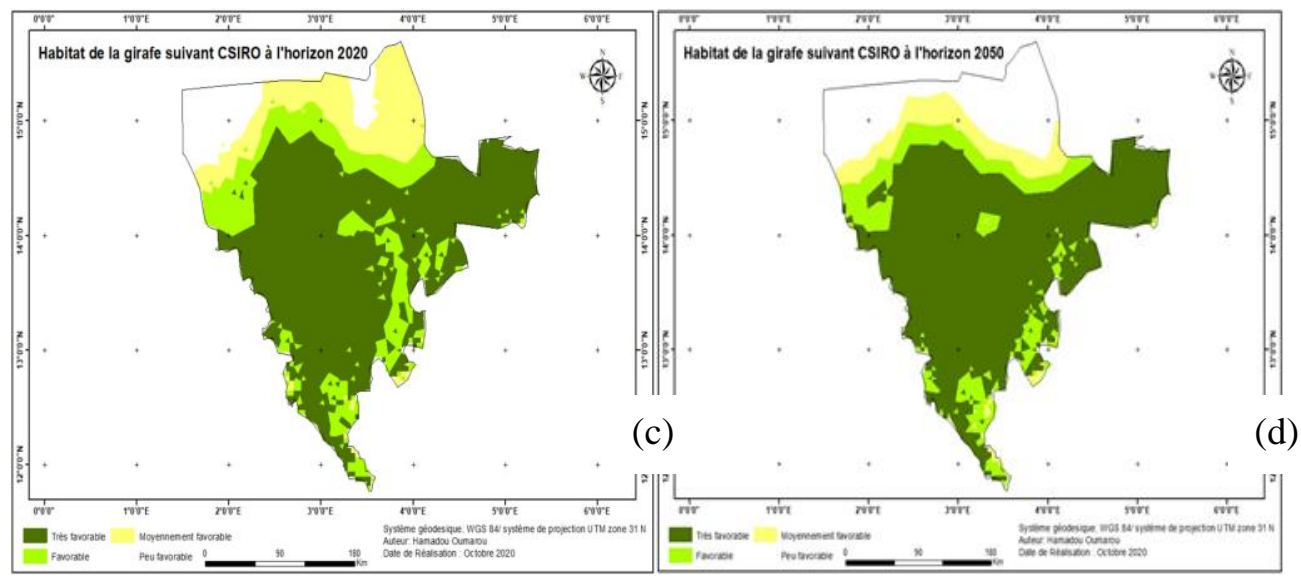

Figure 6: Habitats de la girafe suivant le modèles CSIRO 2020 (c) et CSIRO 2050 (d).

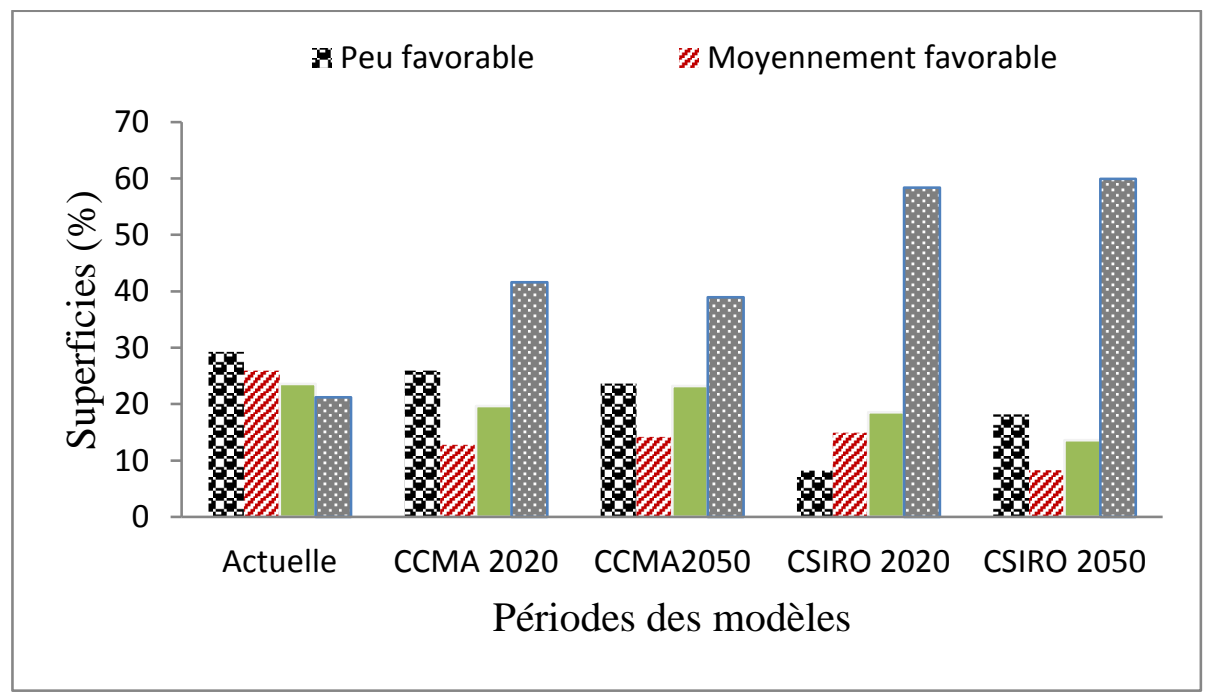

Figure 7 : Evolution des habitats de la girafe suivant les périodes actuelles à CSIRO 2050.

Tableau 2 : Dynamique de l'habitat de la girafe de 1950 à 2050 selon le modèle.

\begin{tabular}{|c|c|c|c|c|c|c|c|c|}
\hline \multirow[t]{2}{*}{ Habitats } & \multicolumn{2}{|c|}{ Peu favorable } & \multicolumn{2}{|c|}{$\begin{array}{l}\text { Moyennement } \\
\text { favorable }\end{array}$} & \multicolumn{2}{|c|}{ Favorable } & \multicolumn{2}{|c|}{ Très favorable } \\
\hline & $\begin{array}{l}\text { Sup } \\
\left(\mathrm{km}^{2}\right)\end{array}$ & \%occupé & $\begin{array}{l}\text { Sup } \\
\left(\mathrm{km}^{2}\right)\end{array}$ & \%occupé & $\begin{array}{l}\text { Sup } \\
\left(\mathrm{km}^{2}\right)\end{array}$ & \%occupé & $\begin{array}{l}\text { Sup } \\
\left(\mathrm{km}^{2}\right)\end{array}$ & \%occupé \\
\hline Actuelle & 24456,81 & 29,29 & 21645,14 & 25,92 & 19659,42 & 23,54 & 17718,81 & 21,22 \\
\hline $\begin{array}{l}\text { CCMA } \\
2020\end{array}$ & 21646,43 & 25,93 & 10678,85 & 12,79 & 16389,59 & 19,63 & 34765,25 & 41,64 \\
\hline $\begin{array}{l}\text { CCMA } \\
2050\end{array}$ & 19736,94 & 23,64 & 11877,68 & 14,22 & 19373,79 & 23,2 & 32491,77 & 38,92 \\
\hline $\begin{array}{l}\text { CSIRO } \\
2020\end{array}$ & 6854,69 & 8,21 & 12444,25 & 14,9 & 15463,77 & 18,52 & 48717,45 & 58,35 \\
\hline
\end{tabular}




\section{DISCUSSION}

Les modifications des formations naturelles dues à la variabilité du climat, ont affecté de façon sensible la survie de la faune sauvage (Ciofolo, 1995 et Morou, 2010). Les températures en Afrique de l'Ouest, et particulièrement dans le Sahel, ont évolué plus rapidement que la tendance mondiale, avec des augmentations allant de $0,2^{\circ} \mathrm{C}$ à $0,8^{\circ} \mathrm{C}$ par décennie depuis la fin des années 1970 dans les zones sahélo sahélienne et soudanienne (CEDEAO, 2008). Cette évolution de la température augmentera dans le temps avec des écarts importants et engendrera sans nul doute des perturbations de plus en plus prononcées dans l'aire de répartition de la girafe (Hamadou, 2014). Ainsi, les besoins en eau de la girafe pourraient devenir plus importants en période de grandes chaleurs. La girafe fera moins de pâture et se déplacera davantage pour la recherche d'eau. Les sècheresses deviennent de plus en plus récurrentes (Giannimi, 2003) et ont pour conséquences une perte significative de la biodiversité. Les contraintes climatiques sont à l'origine d'impacts écologiques sur les écosystèmes des zones arides et semi-arides. En 1982, la girafe était répartie seulement dans les plateaux de Kouré et les vallées du Dallol Bosso Nord. La zone de distribution de la girafe qui était de $840 \mathrm{~km}^{2}$ en 1995 est passée à 3616,243 km² en 2009 (Morou, 2010). Aujourd'hui, l'aire de la girafe est de près de $80.000 \mathrm{~km}^{2}$. La pluviométrie est l'un des principaux facteurs déterminant la distribution de la végétation en zone tropicale (Saadou, 1990 et Mahamane, 2012). Ainsi, la présence de la girafe dans la partie sud (Falmey, Gaya), peut être liée à la pluviométrie. Toutefois, les mouvements ou les déplacements du troupeau de la girafe du noyau central vers certaines zones excentrées ou colonisées, semblerait supposer une influence d'autres facteurs telle que la pression humaine. Aussi, les principales activités humaines constituent des facteurs clés de dégradation des ressources forestières. L'exploitation incontrôlée de l'habitat de la girafe amplifie les impacts de la variabilité climatique sur les ressources (Inoussa, 2011).

Dans ce contexte où, des menaces sur la survie du troupeau et de dégradation de l'habitat pèsent sur la girafe, comprendre les processus écologiques qui régissent les exigences et le comportement de la girafe est une nécessité d'importance croissante. Il s'agit de se faire une idée sur l'utilisation de l'habitat, les couloirs de passage, les tendances de colonisation de l'espace afin de mieux caractériser les futures zones d'expansion. Cela permettra également d'identifier des zones prioritaires de conservation et de prédilections de la girafe d'où l'utilisation des modèles climatiques sur la distribution de la girafe sur plusieurs années.

La modélisation de la distribution des espèces est d'une grande importance en écologie et les modèles peuvent être considérés comme une vue simplifiée de la réalité (Elith, et al., 2006 ; Schmidt et al., 2008 ; Abdou, 2016). Les variables bioclimatiques Bio1 ou Température moyenne annuelle et la Bio 12 ou les précipitations annuelles ont le plus contribué à la prédiction du modèle. La distribution actuelle, c'est-à-dire de 1950 à 2000, a montré un habitat peu favorable pour la girafe. On a noté une faible présence de la girafe dans cet habitat. Cela pourrait être une insuffisance des sources de données sur la présence de la girafe et les missions de terrain dans ce travail n'ont pas visité cette zone à cause de l'insécurité. Cela pourrait être une limite qui doit être interprété avec précautions, comme l'a suggéré (Ndayishimiye, 2011 et Abdou, 2016). Le manque de variable climatique, pour apprécier la pression anthropique dans le modèle est un handicap. Néanmoins, les différentes zones ou localités de présence de la girafe ou susceptible d'accueillir le troupeau de la girafe, ont été visitées. La superficie de l'habitat «très favorable » était la plus petite. Cependant, pour les distributions futures, l'habitat «très favorable ", s'est beaucoup améliorée (21,22 à $59,95 \%)$. Ce qui montre que, la girafe serait plus fréquemment observée dans cette aire de répartition. Ces résultats sont conformes à ceux trouvés par (Yahou, 2020) sur la Modélisation de la distribution des habitats de quelques bovidés, proies des lions des savanes (Panthera leo, Linnaeus 1758) dans la Réserve de Biosphère Transfrontalière du $\mathrm{W}$, Afrique de 
l'Ouest, où Les distributions futures (2020 et 2050) ont montré des habitats très favorables pour l'hippotrague avec une tendance $+129,39$ à $+166,31 \%$ par rapport à la distribution actuelle (1950 - 2000). Les précipitations et les températures contribuent le plus au développement du modèle et expliquent la grande distribution de la girafe. Ce résultat semble corroborer les résultats trouvés par Guisan (2007) où ce sont les paramètres directs, tels que la température et les précipitations, qui affectent le plus la distribution des espèces lorsque la modélisation concerne un large territoire. La valeur de l'AUC $(0,989)$, plus proche de son maximum (1) que de son minimum $(0,5)$ correspondant à une prédiction aléatoire, indique une bonne performance du modèle (Swets, 1988 et Phillips, 2004).

\section{Conclusion}

La modélisation de la distribution est un moyen très pertinent pour définir l'étendue géographique des aires favorables de la girafe du Niger et pour identifier les variables environnementales qui affectent la distribution de son habitat. La carte de l'aire de répartition de la girafe développée dans le cadre de cette étude va améliorer le niveau des connaissances et permettra de mieux appliquer la planification de la conservation et de la gestion de cette espèce. La compréhension des facteurs qui limitent sa distribution spatiale reste une étape très importante pour améliorer les stratégies de gestion et de conservation. Les modèles ont montré une augmentation de plus en plus des superficies de l'habitat très favorable pour la girafe. Le modèle de la distribution a été déterminé par la température moyenne annuelle et les précipitations annuelles et a montré une distribution plus poussée vers les anciens habitats (Tahoua, Maradi) et la partie Sud du pays (Gaya). Le modèle de distribution développé pour la girafe peut aider à choisir de futures zones de distribution où les conditions bioclimatiques sont les plus favorables pour la sauvegarde de l'espèce, ce qui représente une solution pour réduire la pression sur les espèces végétales consommées, tout en développant des mesures pour une cohabitation harmonieuse avec les communautés locales et éviter la migration du troupeau de la girafe vers des zones où elles seront hors contrôle.

\section{CONFLIT D'INTERETS}

Les auteurs de ce manuscrit déclarent qu'il n'y a aucun conflit d'intérêt entre eux.

\section{CONTRIBUTIONS DES AUTEURS}

OAA, YH et MB ont contribué à la rédaction puis à l'amélioration du manuscrit. AM a supervisé les travaux depuis le protocole jusqu'à l'aboutissement du manuscrit. Il a également apporté des conseils pour la collecte des données. Tous les auteurs ont approuvé la version finale du manuscrit.

\section{REMERCIEMENTS}

Nous exprimons notre gratitude aux populations locales des communes visitées, à l'Association pour la Sauvegarde de la Girafe du Niger (ASGN) et le Bioparc de Doué La Fontaine (France).

\section{REFERENCES}

Abdou L. 2016. Importance ethnobotanique, dynamique des peuplements et écologie de Prosopis africana (G. et Perr) Taub. $\mathrm{Au}$ Niger. Thèse de Doctorat de l'université Dan Dicko Dankoulodo de Maradi-Niger, p.157.

Ambouta JMK. 1997. Définition et caractérisation des végétations d'une brousse tigrée de l'Ouest nigérien: Fonctionnement et gestion des écosystèmes forestiers sahéliens. John Libbey Eurotext. Paris : 41-57.

Ambouta JMK. 1999. Etat des lieux sur les connaissances en matière d'habitat pour les girafes du Niger, p.12.

Ambouta JMK.1994. Etude des facteurs de formation d'une croûte d'érosion et de ses relations avec les propriétés internes d'un sol sableux fin au Sahel. Ph.D, Universitaire Laval, Québec, p.97.

Austin MP. 2007. Species distribution models and ecological theory: a critical assessment and some possible new approaches. Ecol Model., 200: 1-19. DOI: 10.1016/j.ecolmodel.2006.07.005 
Baldwin RA. 2009. Use of maximum entropy modeling in wildlife research. Entropy, 11(4): 854-866. DOI:10.3390/e11040854

Barbosa AM, Real R, Vargas JM. 2009. Transferability of environmental favourability models in geographic space: the case of the iberian desman (galemys pyrenaicus) in Portugal and Spain. Ecol Model, 220: 747-754. DOI: 10.1016/j.ecolmodel.2008.12.004

Benabid A. 2000. Flore et Ecosystèmes du Maroc. Évaluation et Préservation de la Biodiversité. Ibis Press. Paris - Librairie et éd. Kalila Wa Dimna. Rabat. 1 vol; 338-343.

\section{CEDEAO-CLUB/SAHEL/OCDE/CILSS,} Climate and Climate Change. 2008. The Atlas on Regional Integration in West Africa.

Ciofolo I, LE Pendu Y. 1998. Les girafes du Niger, de l'analyse éthologique au développement local, Niamey, UE (Union Européenne), SNV, p. 73.

Ciofolo I. 1995. West Africa's last giraffes: the conflict between development and conservation. J. Trop. Ecol, 11: 577-588.

Cornelis D. 2011. Ecologie du déplacement du buffle de savane ouest-africain (Syncerus caffer brachyceros). Thèse de Doctorat de l'université de Montpellier 2 (France), p. 290.

Creel S, Winnie Jr J, Maxwell B, Hamlin K, Creel M. 2005. Elk alter habitat selection as an antipredator response to wolves. Ecology, 86: 3387-3397.

Dormann, Elith J, Bacher S, Buchmann C, Carl G, Carre G, Lautenbach S, Collinearity. 2013. A review of methods to deal with it and a simulation study evaluating their performance. Ecography, 36(1) : $27-46$.

Elith J, Leathwick JR. 2009. Species distribution models: Ecological explanation and prediction across space and time. Annu. Rev. Ecol. Evol. Syst, 40: 677-697.

Elith J, Graham CH, Anderson RP, Dudik M, Ferrier S, Guisan A, Hijmans RJ, Huettmann F, Leathwick JR, Lehmann A, Li J, Lohmann LG, Loiselle BA, Manion G, Moritz C, Nakamura M, Nakazawa Y,
Mcc J, Overton, Peterson AT, Phillips SJ, Richardson KS, Scachetti-Pereira R, Schapire RE, Soberon J, Williams S, Wisz MS, Zimmermann NE. 2006. Novel methods improve prediction of species' distributions from occurrence data. Ecography, 29: 129-151.

Fandohan B, Gouwakinnou GN, Fonton NH, Sinsin B, Liu J. 2013. Impact des changements climatiques sur la répartition géographique des aires favorables à la culture et à la conservation des fruitiers sous-utilisés : cas du tamarinier au Bénin. Biotechnol. Agron. Soc. Environ, 17(3) : 450-462.

Fawcett T. 2006. An introduction to roc analysis. Pattern Recognit. Lett, 27: 861874.

Feeley KJ, Silman MR. 2011. The data void in modeling current and future distributions of tropical species. Glob. Chang. Biol, 17: 626-630.

Fleishman E, Macnally R, Fay JP, Murphy DD. 2001. Modeling and predicting species occurrences using broad-scale environmental variables: an example with butterflies of the great basin. Conserv. Biol, 15: 1674- 1685.

Frankline J. 2009. Mapping Species Distributions: Spatial Inference and Prediction. Cambridge University Press: Cambridge.

Giannimi A, Saravanan R, Chang P. 2003. Oceanic of Sahel rainfall on interannual to interdecadal time scales. Science, 302: 1027-1030.

Guisan A, Zimmermann NE. 2000. Predictive habitat distribution models in ecology. Ecol. Model, 135: 147- 186.

Guisan A, Graham CH, Elith J, Huettmann F. 2007. Nceas Species Distribution Modelling Group, Sensitivity of predictive species distribution models to change in grain size. Divers. Distrib, 13: 332-340.

Hamadou O. 2009. Analyse des relations entre la population des girafes et quelques attributs vitaux de son habitat, mémoire ITA, Faculté d'Agronomie de Niamey, p. 46. 
Hamadou O. 2014. Variabilités climatiques et conservation de la girafe d'Afrique de l'Ouest (Giraffa camelopardalis peralta) au Niger. Mémoire de Master 2 en Gestion des Ressources Naturelles et de la Biodiversité, Faculté des Sciences Agronomiques, Université d'AbomeyCalavi du Bénin, p.81.

Hanley JA, Mcneil B. 1982. The Meaning and use of the area under a receiver operating characteristic (ROC). Curve Radiology, 143: 29-36.

Hijmans RJ, Cameron SE, Parra JL, Jones PG, Jarvis A. 2005. Very high-resolution interpolated climate surfaces for global land areas. Int. J. Climatol, 25(15) : 19651978.

Inoussa MM. 2011. Dynamique des forêts claires dans le Parc national du W du Niger, these de doctorat, université Abdou Moumouni, spécialité, biologie et écologie végétales, p. 91.

IPPCC, Field CB, Barros VR, Dokken DJ, Mach KJ, Mastrandrea MD, Bilir TE, Chatterjee M, Ebi KL, Estrada YO, Genova RC, Girmab, Kissel ES, Levy A, Maccracken S, Mastrandrea PR, White LL. 2008. Climate change 2014, impacts, adaptation and vulnerability part: a global and sectorial aspects. Contribution of working group ii to the fifth assessment report of the intergovernmental panel on climate change. Cambridge University Press, Cambridge, United Kingdom and New York, Ny, USA.

Joppa LN, Loarie SR, Pimm SL. 2008. On the protection of "protected areas". Proceedings of the National Academy of Sciences, 105: 6673.

Kumar S, Stohlgren TJ. 2009. Maxent modelling for predicting suitable habitat for threatened and endangered tree canacomyrica monticola in New Caledonia. J. Ecol. Nat. Environ., 1(4): 94-98.

Mahamane A, Morou B, Zaman-Allah M, Saadou M, Saley K, Bakasso Y, Wata IS, Oumani AA, Jauffret S. 2012. Climate Variability in Niger: Potential Impacts on vegetation distribution and productivity.
Journal of Environmental Science and Engineering, B1 : 49-57.

MDCE. 2016. Troisième communication nationale à la CCNUCC. Ministère délégué auprès du Ministre de l'Energie, des Mines, de l'Eau et de l'Environnement, chargé de l'Environnement, Maroc.

Miller RG. 1974. The Jackknife a Review. Biometrika, 61: 1-15.

Morou B, Ambouta JMK, Mahamane A, Zaman-Allah M, Sadou M, Sinsin B. 2009a. Interaction entre hommes et girafes dans la zone de distribution de la girafe au Niger. Annales de l'Université Abdou Moumouni, Tome X-A : 75-86.

Morou B. 2010. Impacts de l'occupation des sols sur l'habitat de la girafe au Niger et enjeux pour la sauvegarde du dernier troupeau de girafes de l'Afrique de l'Ouest. Thèse pour obtenir le grade de Docteur de l'Université Abdou Moumouni de Niamey (Option : Biologie Appliquée), p. 198.

Moukrim S. 2018. Modélisation de la distribution spatiale des espèces endémiques pour leur conservation : cas de L'Argania Spinosa (L.) Skeels. Revue d'Ecologie (Terre Et Vie), 73(2) : 153166.

Ndayishimiye J. 2011. Diversité, endémisme, géographie et conservation des Fabaceae de l'Afrique Centrale. Thèse présentée en vue de l'obtention du Diplôme de Docteur en Sciences. Université Libre de Bruxelles, p.269.

Padalia H, Srivastava V, Kushwaha SPS. 2014. Modeling potential invasion range of alien invasive species hyptis suaveolens (L.) Poit. In India: Comparison of Maxent and Garp. Ecol. Inform, 22: 36-43.

Peterson AT, Soberon J. 2012. Species distribution modeling and ecological niche modeling: getting the concepts right. Nat. Conservação, 10: 102-107.

Phillips SJ, Anderson PR, Schapire RE. 2006. Maximum entropy modeling of species geographic distributions. Ecol Model, 190(3) : $231-259$. 
Ripple WJ, Beschta RL. 2004. Wolves and the ecology of fear : can predation risk structure ecosystems? BioScience, 54: 755-766.

Saadou M. 1990. La végétation des milieux drainés nigériens à l'Est du fleuve Niger : Thèse de Doctorat ès - Sciences Naturelles. Université de Niamey, p. 395.

Sampoux JP, Badeau V. 2009. Modélisation de La niche écologique des fétuques à feuilles fines: Quels apports pour la conservation et la valorisation des ressources génétiques? Innov. Agron, 7: 79-91.

Schmidt M, König K, Müller JV. 2008. Modelling species richness and life form composition in Sahelian Burkina Faso with remote sensing data. Journal of Arid Environments, 72(8): 1506-1517.
Scott JM, Heglund PJ, Morrison ML, Haufler JB, Raphael MG, Wall WA, Samson FB. 2002. Predicting Species Occurrences: Issues of Accuracy and Scale. Island Press: Washington, DC.

Swets JA. 1988. Measuring the accuracy of diagnostic systems. Science, $240: 1285-$ 1293.

Yahou H., Oumani AA, Abdou L, Mahamane A. 2020. Modélisation de la distribution des habitats de quelques bovidés, proies des lions des savanes (Panthera leo, linnaeus, 1758) dans la réserve de Biosphère Transfrontalière du $\mathrm{W}$, Afrique de l'Ouest, Université de Diffa, Niger. Afrique Science, 17 (1) : 177-188. http:// www.worldclim.org, consulté le 13 mai 2021 à 16 heures 41 minutes. 